\title{
Towards Autonomous Locomotion: Slithering Gait Design of a Snake-like Robot for Target Observation and Tracking
}

Conference Paper · September 2017

DOI: $10.1109 /$ IROS.2017.8206095

CITATIONS

0

7 authors, including:

\section{Zhenshan Bing}

Technische Universität München

9 PUBLICATIONS 6 CITATIONS

SEE PROFILE

\section{Guang Chen}

Technische Universität München 19 PUBLICATIONS 77 CITATIONS

SEE PROFILE
READS

34

Kai Huang

University of Science and Technology Beijing

94 PUBLICATIONS 544 CITATIONS

SEE PROFILE

Florian Röhrbein

Technische Universität München

84 PUBLICATIONS 281 CITATIONS

SEE PROFILE

Some of the authors of this publication are also working on these related projects: 


\title{
Towards Autonomous Locomotion: Slithering Gait Design of a Snake-like Robot for Target Observation and Tracking
}

\author{
Zhenshan Bing ${ }^{1}$, Long Cheng ${ }^{1}$, Kai Huang ${ }^{2}$, Zhuangyi Jiang ${ }^{1}$, \\ Guang Chen ${ }^{1}$, Florian Röhrbein ${ }^{1}$, and Alois Knoll ${ }^{1}$
}

\begin{abstract}
In this paper, a biologically inspired 3D slithering gait for a snake-like robot is designed and implemented for the purpose of target tracking. First, by balancing the forward speed and the stability of the robot, a straight slithering gait is modelled, under which the robot can march straight, fast, and stably. Then, for the purpose of steering, the straight slithering gait is modified into a biased slithering gait. The relationship between turning radius and gait parameters is analyzed by the resistive force theory. With the head composition algorithm, we investigate the orientation problem of the snake robot's head module to obtain stable visual information during the locomotion process. Finally, with the guidance of the vision sensor mounted in the head module, target tracking simulations and prototype experiments are conducted to demonstrate the practicality and effectiveness of the slithering gait in autonomous locomotion scenarios.
\end{abstract}

\section{INTRODUCTION}

With the increasing demand of autonomy and self-adaptive behavior, snake-like robots are required not only to move efficiently, but also autonomously [1]. Specifically, robots are capable of avoiding the obstacles by themselves and navigating to the target without remote control by operators. Snake-like robots with autonomous locomotion capabilities will have significant improvement on practical implementations, such as disaster rescue, military surveillance, and factory maintenance [1].

Currently, a number of snake-like robots can achieve diverse locomotion, for instance, serpentine forward [2], roll to the sideways [3], climb along poles, and swim under water [4]. However, none of those snake-like robots can achieve autonomous locomotion, i.e., the capabilities to autonomously decide how, when, and where to move. Snakelike robots achieve effective 3D locomotion by twisting their bodies heavily. Therefore, they can hardly obtain stable and effective images to guide the locomotion, unlike vehicles or legged robots on which the mounted cameras or lidar are relatively stable. This key issue brings us the challenge: how to design a gait that can make the snake robot move effectively, and meanwhile obtain useful visual information.

For snake-like robots, there have been several gaits adopted to achieve diverse locomotion. For instance, snakelike robots can move smoothly on the flat ground under serpentine gait, but suffer the locomotion limitation in 3D space or uneven terrain. The robots can move to the sideways quickly and overcome small obstacles under rolling and sidewinding gaits. Since the robot is rotating itself under these gaits, it will fail to gather stable and useful visual information. Moreover, the moving direction is not aligned with the direction of the view field, which is not biologically

Authors Affiliation: ${ }^{1}$ Technical University of Munich, Department of Informatics, ${ }^{2}$ Sun Yat-Sen University.

Email: $\{$ bing, chengl, huangk, jiangz, guang, roehrbei, knoll $\} @$ in.tum.de

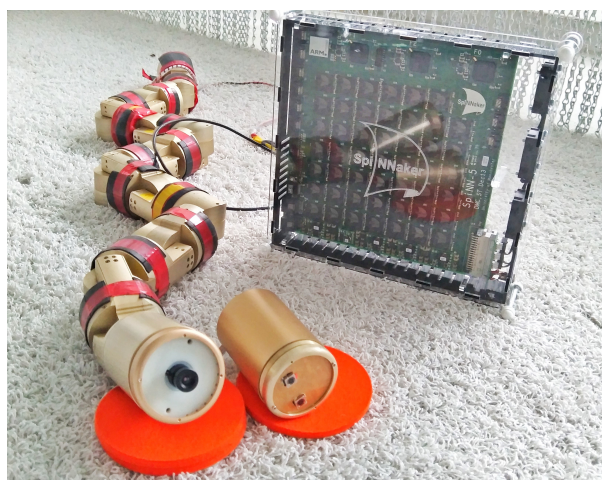

Fig. 1: Left: the snake-like robot equipped with stereo cameras/embedded dynamic vision sensor (eDVS) [5]. Right: The SpiNNaker board [6].

reasonable. Thus, these gaits are not suitable for autonomous locomotion.

Unlike those aforementioned gaits, slithering is a forward locomotion gait where the snakes use undulations to push their bodies forward [7]. A snake can march forward quickly under this gait. More importantly, the snake head can still remain stable to locate the moving direction of the quarry or the natural enemies. Therefore, slithering is a promising gait for snake-like robots to achieve autonomous locomotion.

The slithering gait has been investigated for wheeled or wheel-less snake robots $[8,9,10]$. Wu [9] designed a neural controller by adding sensory inputs to achieve self-adaptive steering for collision-free behavior. Tanaka [10] adopted a curve-based controller for self-collision avoidance, meanwhile maintaining the head position and orientation. However, these works are not further adopted for autonomous locomotion. The reasons are manifold. First, since the snake robot stretches its body along the direction from the head module to the tail module, the robot is prone to rotating sideways. Unlike a real snake with abundant arthrosis, the number of contact points for the snake-like robot to touch the ground is also limited. These reasons may cause dynamic instability and fail the locomotion. Second, the redundant locomotion mechanism of snake-like robot may cause undesired frictional drag that influences the movement. Thus, it's difficult to steer accurately and agilely when the robot needs to change its direction. Finally, even the orientation and twisting of slithering gait are much less than other gaits, the head module still has fatal orientation problem which is insufficiently stable to gather useful visual images.

This paper focuses on aforementioned issues and addresses the slithering gait design for autonomous locomotion purpose. The contributions of this work are summarized as follows. 


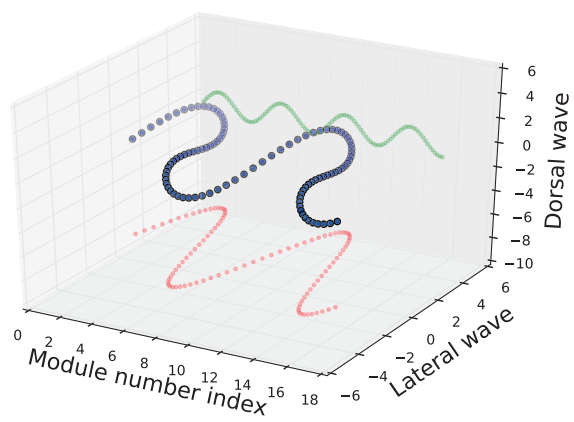

(a) Backbone curve without the linear reduction

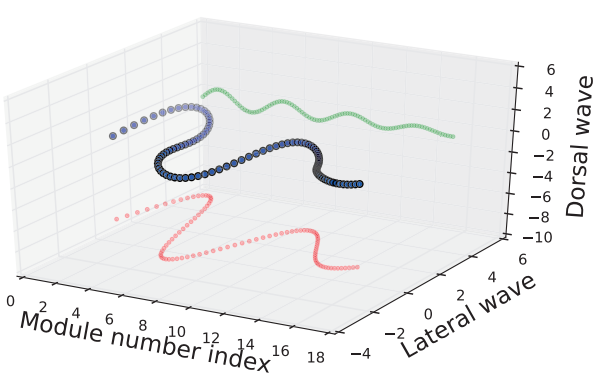

(b) Backbone curve with the linear reduction

Fig. 2: In both figures, the blue scatter in 3D plane is the backbone of the snake robot when it slithers. The red and green scatters are the projection of the backbone in the lateral and dorsal planes.

- Inspired by real snakes, a slithering gait for the snakelike robots is designed and modeled for autonomous locomotion purpose. The relationship between the kinematic characteristics and the gait parameters are analyzed in detail, including the stability, forward velocity, and steering radius.

- An orientation compensation algorithm is proposed to decrease the twisting of the head module when the robot slithers forward. As a result, the yaw angle is decreased by $50 \%$ compared with the unadjusted locomotion.

- Extensive simulations and prototype experiments are conducted for target tracking and following scenarios. The results prove the practicality and effectiveness of the slithering gait in autonomous movement scenarios.

The rest of this paper is organized as follows: Section II presents related work on the gait design for snake-like robots. The slithering gait is modeled and analyzed in Section III. The head orientation compensation algorithm is proposed in Section IV. In Section V, simulations and experiments results supporting our theoretical findings are presented. Section VI concludes this paper and presents the future work.

\section{BACKGROUND}

Unlike other land animals with legs, snakes achieve diverse locomotion by twisting their bodies on various terrain, such as desert, swamps, and water. Those cyclic twisting motions helping snakes achieve different locomotion capabilities, are called gaits [3]. There are four common gaits for most snakes, i.e., the serpentine gait, reticular gait, sidewinding gait, and concertina gait [1]. Inspired by those moving patterns, researchers developed several biological gaits for snake-like robots to move inside and outside door environment [3, 11]. Serpenoid curve [12] was the first effective approach adopted to control snake-like robot by imitating real snake movement. However, this gait is mainly suitable for snake-like robots with passive wheels [1], which limits those robots to move only over flat ground.

To cope with this issue, snake-like robots with 3D locomotion capabilities were developed, accompanying with 3D gaits like rolling [13] and sidewinding [14]. Rolling is used for climbing poles, crawling inside pipes, or moving the snake robot sideways [3]. Zhen [13] modeled the rolling gait by changing its rolling shape to cross obstacles. Sidewinding is another efficient gait for locomotion over flat ground. Hatton [15] identified the stability of sidewinding and its conditions for sidewinding on the slope. Furthermore, Gong $[16,17,18]$ modified the gait for steering purpose by transforming the amplitude of the gait equation [3] into a function depending on the element index. Although these 3D gaits enhanced the locomotion ability of snake-like robots, it is still far from autonomous locomotion. One of the key features for autonomy is the visual guidance from sensors [19], however these two gaits can not supply stable visual information due to the consistent body twisting.

Slithering is a linear locomotion gait adopted by limbless terrestrial animals, by sliding their bodies along the ground [7]. The snake pushes the points where the body bends and that proposes sideways. When two opposite coils push at the same time, the side-way force cancels each other out and the snake propels forward. Some snakes [7] can move fast using this gait and the head can remain stable during the locomotion, where locates the visual system for real snake. Therefore, slithering is expected as a promising gait for autonomous locomotion of snake-like robots. Further research about the snakes movement was made by $\mathrm{Hu}$ [7] who focused on the slithering of legless animals. From the marks snakes leave on the sand, it is clear that the snake only push the points where the body bends. The shape of those marks are very similar to the scratches on the ice surface from the skate blades. He also demonstrated that snakes push themselves forward by lifting parts of their body slightly off the ground when moving. This behavior can effectively avoid the undesired drag friction force and apply greater pressure to push against the ground. All these biological findings will enlighten the research about the locomotion of the snake-like robots.

\section{Modeling of Slithering Gait}

The purpose of motion modeling is to analyze the kinematics characteristics and generate control signals for snake-like robots to achieve the desired locomotion.

\section{A. Gait Equation}

Gait equation [3] is a concise expression for gaits of a snake-like robot. The gait equation is divided into two wave functions, one for the lateral plane and one for the dorsal plane. Each wave function has a dependency on time and space. Since the robot consists of finite modules, the space has to be discrete to the number of elements. Comparing to other gaits such as sidewinding and rolling, the slithering 
gait has a small support area. The robot is prone to rotate sideways when using this gait. Therefore, the challenge for designing slithering gait is how to maintain the stability of the robot.

A modified and extended gait equation is proposed to shape the robot body into a more stable geometry, described as,

$$
\begin{gathered}
\alpha(n, t)_{\text {odd }}=C_{\text {odd }}+P \cdot A_{\text {odd }} \cdot \sin \left(\Omega_{\text {odd }} \cdot n+\omega_{\text {odd }} \cdot t\right) \\
\alpha(n, t)_{\text {even }}=C_{\text {even }}+P \cdot A_{\text {even }} \cdot \sin \left(\Omega_{\text {even }} \cdot n+\omega_{\text {even }} \cdot t+\delta\right) \\
P=\left(\frac{n}{N} \cdot z+y\right) \in[0,1], \quad \forall n \in[0, N]
\end{gathered}
$$

All the explanations of the symbols in the gait equations are listed in Table I.

TABLE I: Descriptions of the extended gait equations

\begin{tabular}{ll}
\hline Items & Descriptions \\
\hline$\alpha_{\text {even }}, \alpha_{\text {odd }}$ & Joints commands in lateral and dorsal planes \\
\hline$C_{\text {even }}, C_{\text {odd }}$ & Body shape offset in lateral and dorsal planes \\
\hline$A_{\text {even }}, A_{\text {odd }}$ & Amplitude in lateral and dorsal planes \\
\hline$\Omega_{\text {even }}, \Omega_{\text {odd }}$ & Spatial frequency in lateral and dorsal planes \\
\hline$\omega_{\text {even }}, \omega_{\text {odd }}$ & Time frequency in lateral and dorsal planes \\
\hline$N$ & Module numbers \\
\hline$P$ & Linear dependency \\
\hline$x$ & Cycle numbers \\
\hline$n$ & Module subscript \\
\hline$\delta$ & Phase difference \\
\hline
\end{tabular}

\section{B. Contact Points}

Contact points are the interaction parts between the snakelike robot and the ground, which generate the propulsion to move. Since the snake robot slithers forward with an S-like shape, the stability against the rolling tendency to the side is important. For a stable locomotion, at least three contact points are required during the locomotion process.

As shown in Fig. 2(a), the blue scatter in 3D plane is the backbone of the snake robot when it slithers. The contact points are the minimum values of the wave in the dorsal plane. We can see that all the contact points are uniformly distributed at both sides of the backbone curve of the robot. The number of the contact points is decided by the cycle numbers of the wave in the dorsal plane, which is related to the $x$ in (1). If $x=1$, there will be one cycle wave in the dorsal plane and one contact point.

When real snakes slither forward, they swing their bodies in the edge to push against the ground, which can generate the largest thrust force. To imitate the motions of real snakes, the robot also should use the body edges to touch and push against the ground. Thus, for the body curve of a snakelike robot, the minimum points in the dorsal plane should be corresponding to the extremum points in the lateral plane, which indicates the contact points of the robot body and the ground. The frequency of the projection wave in the lateral plane should be half of the wave in dorsal wave. We can conclude that,

$$
x_{\text {dorsal }}=2 \times x_{\text {lateral }}
$$

To ensure at least three contact points during the locomotion process, the dorsal wave should have three minimum points. This means $x_{\text {lateral }} \geq 1.5$.

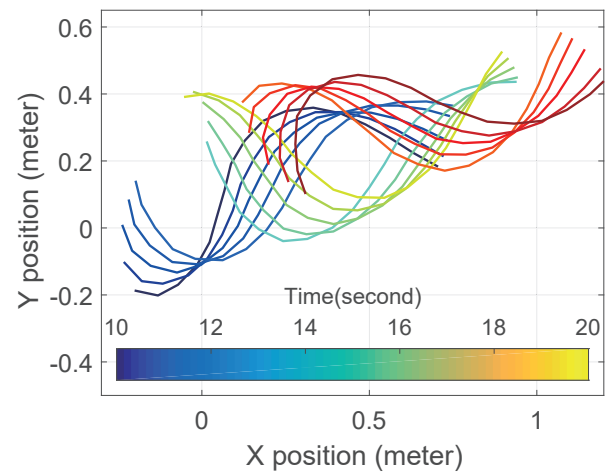

Fig. 3: Slithering pattern of the snake robot. Color indicates the montage time.

\section{Linear Reduction}

Combined the dorsal and lateral waves, the shape of the snake-like robot body should be like a cylinder. Although this shape can drive the robot to move forward, it is still insufficient self-adaptive for some tasks, such as surveillance and target tracking tasks. To meet these requirements, the stabilization of the head module and its sensors should be maintained. Therefore, the shape of this locomotion shall be changed from a cylinder to a frustum, as shown in Fig. 2(b).

The linear reduction equation is shown in (1). The amplitude for each module is defined as a linear function dependent on the module number. The linear coefficient starts at $y$ for the head module and ends at $z$ for the tail module. Due to the reduction to a frustum shape, the head module has a small amplitude and swings less to maintain stable. The linear dependency to the module numbers denoted by $P$, can be also changed into any other polynomial functions. Different reduction methods will cause different body shape in the lateral plane. The slithering pattern is shown in Fig. 3, which indicates that the body curve swings forward over time.

\section{Biased Slithering}

Biologists used X-ray to study the undulation pattern of limb-less animals. The results revealed that the body undulation pattern of the snake was well approximated by a planar sinusoidal wave that propagated from the head to the tail, while the center of mass moved forward in approximately the horizontal plane [20]. In their research, Resistive Force Theory (RFT) was adopted to analyze the swimming movement of animals in fluid or granular media, like the dry desert or muddy environment. By segmenting the object body into a series of chain-connected elements and analyzing their normal and tangential force, the undulatory locomotion of those animals can be modeled and predicted.

Inspired by that, we use RFT to analyze the 3D locomotion of our snake-like robot. As shown in Fig 4, the snake-like robot slithers in the lateral wave by propagating a sinusoidal wave from the head to the tail. Take one module as example, the resistive force $d F$ is generated by the friction force from the reaction between each module and the ground. The $d F$ can be divided into $d F_{\perp}$ and $d F_{\|}$along the directions parallel and perpendicular to the forward direction. The net force on 


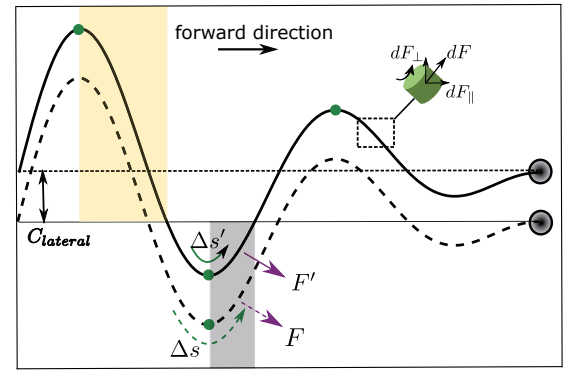

Fig. 4: Illustration of the basic idea behind Resistive Force Theory for slithering forward. The prescribed lateral wave propagates along the body and generate reaction forces to push the robot forward.

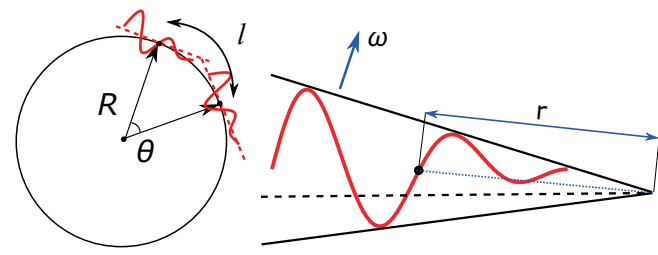

Fig. 5: Left figure is the top view of the turning slithering for a snake robot. Right figure shows the geometric relationship between turning velocity $\omega$ and turning radius $r$.

the snake-like robot is then computed from the integral

$$
\begin{gathered}
F=\int\left(d F_{\perp}+d F_{\|}\right)=f \int_{0}^{s} d s[\vec{n} \cdot \vec{v}+\vec{o} \cdot \vec{v}] \\
F_{\text {forward }}=\int_{0}^{A+C} d F_{\|} d s-\int_{0}^{A-C} d F_{\|} d s
\end{gathered}
$$

where the unit normal vector $\vec{n}$ can be determined from the lateral wave, the unit vector $\vec{v}$ denotes the forward direction and its orthogonal vector is $\vec{o}$. The lateral wave length is denoted by $s$. The amplitude and the bias are presented by $A$ and $C$. Since the serpenoid cure is symmetrical along the forward direction, the side-way force cancels each other out and propels the robot forward. From 4, we can conclude that the forward force $F_{\text {forward }}$ is not correlated with the amplitude bias. According to RFT, the pushing force is decided by the shape of the lateral wave and the frequency of dorsal wave. The rotating speed $\omega$ along the center is in direct proportion to the forward velocity.

Mean value adjustment method (MVA) is one of the straightforward turning methods for snake-like robots who travel in the plane with passive wheels. The MVA method adds an equally amplitude bias term to each joint's commands. This operation directly changes the mean value of the lateral wave, and thus changes the moving direction. As shown in Fig 4, before the mean value adjustment, the lateral curve is symmetrically distributed along the moving direction. The values of $s$ in (3) is equivalent in both sides of the moving directions. Therefore, the left-side-way and rightside-way forces can cancel each other. After the mean value adjustment, the positive part of the body curve is longer than the negative part. It will generate more right-side-way force than the left-side-way force. Therefore, the snake-like robot will turn right compared to the previous moving direction.
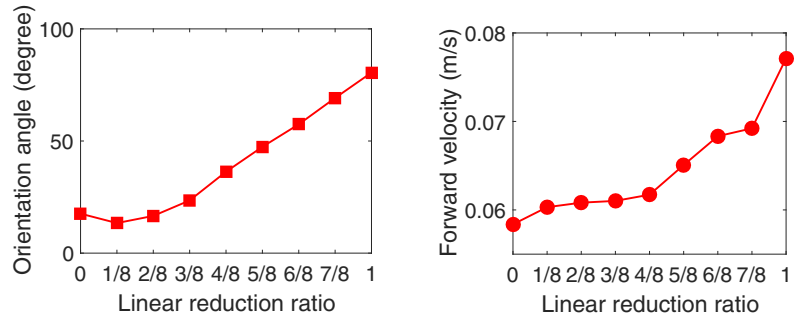

Fig. 6: Left: The relation between the orientation angle and the linear reduction ratio. Right: The relation between the forward velocity and the linear reduction ratio.

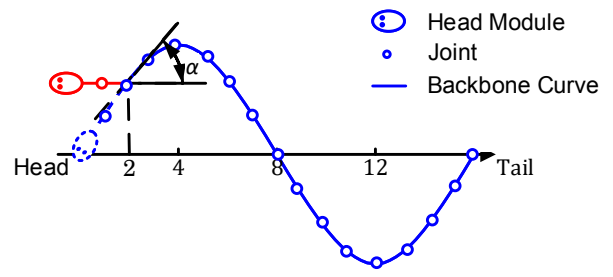

Fig. 7: Top view of the backbone curve of the snake robot.

The relationship between the turning radius and the gait parameters are shown in Fig. 5. The turning radius can be calculated as,

$$
R=\frac{l}{\theta}=\frac{v}{\omega}
$$

where $v$ donates the forward velocity, $\omega$ presents the turning velocity as mentioned before. Since the forward velocity is not correlated to the amplitude bias, in this case, the velocity $v$ can be regarded as a constant. The angular velocity $\omega$ is directly related to the force in the perpendicular direction, which is calculated as,

$$
F_{\text {perpendicular }}=\int_{0}^{A+C} d F_{\perp} d s-\int_{0}^{A-C} d F_{\perp} d s=2 \int_{0}^{C} d F_{\perp} d s
$$

$d F_{\perp}$ is determined by the mass and the friction coefficient of the snake-like robot, therefore, can be treated as a constant. From (6), we can conclude that the force in the perpendicular direction is proportional to the amplitude bias $C$. Combined with (5), we can see that the turning radius $R$ is inversely proportional to the amplitude bias $C$.

\section{HEAd ORIENTATION COMPOSITION}

A rattlesnake can adjust its head posture to look at the direction of locomotion, even under the sidewinding gait. It is important for snakes that they can observe the environment effectively during the locomotion, which is helpful to avoid natural enemies or find the quarry.

Since the snake-like robot slithers forward by swinging its body, this undulatory locomotion inevitably causes the head module swing back and forth. This is not acceptable for further advanced locomotion control based on sensory feedback from the head module, especially the vision sensor inside. Although the linear reduction method could reshape the robot body into a less swing frustum shape from cylinder shape, it is still insufficient for visual sensor, let alone dragging down the forward speed. Simulation results are shown in Fig. 6, the orientation angle decreases with the 


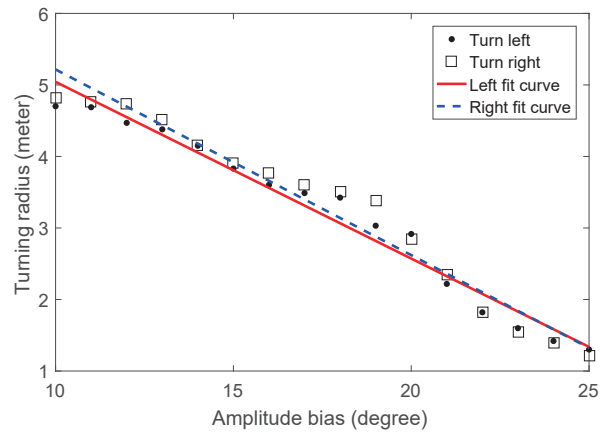

Fig. 8: The relation between the amplitude bias $C$ and the turning radius $R$. The turning experiment results are shown with dot marker and box marker, for turning left and right respectively. The solid line and the dash line describe the linear fitting result for turning left and right.

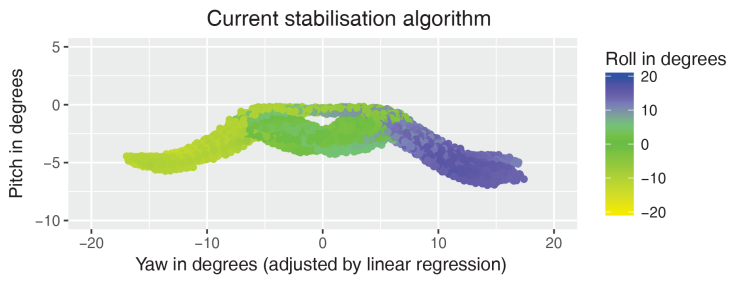

(a) The orientation angles without the compensation algorithm.

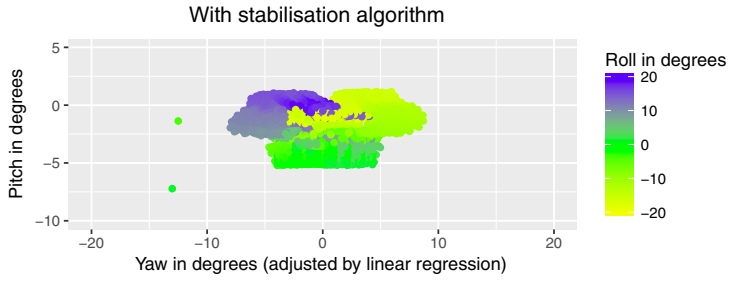

(b) The orientation angles with the compensation algorithm.

Fig. 9: The orientation angles of the head module during the slithering locomotion process.

linear reduction ratio, meanwhile the forward velocity is reduced as well. We know that the snake can travel with the rear part of the body and attack with the front part of the body. Therefore, the first two modules in the dorsal and lateral plane are used to composite the yaw and pitch angle during the slithering locomotion. As shown in Fig. 7, in the lateral plane, the backbone curve is a pre-defined time-varying sinusoid wave, propagating along the snakelike robot. $\alpha$ is the second joint angle, which is donated by (1). $\alpha_{\text {compensate }}$ can be obtained by taking a derivative with respect to time.

$$
\alpha_{\text {compensate }}=\arctan \left(\cos \left(\Omega_{\text {even }} \cdot n+\omega_{\text {even }} \cdot t\right) \cdot \omega_{\text {even }}\right)
$$

where $n=2$. Combined with the pre-set value, we can obtain the command for the second module.

$$
\alpha_{\text {command }}=\alpha(n, t)_{\text {even }}-\alpha_{\text {compensate }}
$$

By giving $\alpha_{\text {command }}$ to the composting joint, the head module could be aligned to the forward direction theoretically. Simulation results are described in detail in Section V.

\section{RESUlts AND Discussions}

For further verification, a mobile target tracking scenario is simulated using Virtual Robot Experimentation Platform. For the prototype experiment, a fixed target approaching scenario is conducted to demonstrate the effectiveness of the proposed slithering gait.

\section{A. Simulations}

In order to demonstrate the turning radius conclusion, a groups of simulation experiments are conducted. The simulation results can be seen in Fig. 8. By varying the amplitude bias from $10^{\circ}$ to $25^{\circ}$, the snake-like robot turns left with a decreasing radius. By varying the amplitude bias from $-10^{\circ}$ to $-25^{\circ}$, the robot turns right with a decreasing radius. The results clearly exhibit that the inverse relationship between the turning radius and the amplitude bias, no matter turning left or right.

Then, a red car tracking the black path on the ground is regarded as the target. Using the proposed slithering gait, a snake-like robot with 16 module keeps observing and tracking the red car during the locomotion, as shown in Fig. 10. The target red car moves along the black circular path on the ground, which can be regarded as random movement for the snake-like robot. A vision sensor is mounted in the head module to obtain the color information of the tracking target. By extracting the relative position of the target and the head module, the snake-like robot can calculate the moving direction to follow the target. As seen from the figure, the snake robot changes its direction with the moving car, meanwhile the visual images are shown in each subfigures.

The head orientation data is recorded during the locomotion process compared with the uncompensated process as shown in Fig. 9. As we can see in Fig. 9(a), the head module swings in a range from $-17^{\circ}$ to $17^{\circ}$ in the horizontal direction. In Fig. 9(b), the yaw angle is clearly decreased by $50 \%$. The range is about from $-8^{\circ}$ to $8^{\circ}$ in the horizontal direction. The results can prove the effectiveness of the orientation compensation algorithm to stabilize the head module.

\section{B. Prototype Experiments}

The snake robot prototype is shown in Fig. 1. Our snakelike robot has a modular design consisting 13 actuated modules and a head module. All the output shafts are alternately aligned with the robot's lateral and dorsal planes to generate 3D locomotion. Each module is connected to the adjacent modules and allows a full $180^{\circ}$ rotation.

At the beginning, the robot forms itself into a stable position and then raises its head to scan the environment to find the target with the help of the neuromorphic vision sensor (eDVS) mounted on the head module, as shown in Fig. 11(a), at $t=5 \mathrm{~s}$. After finding the target, the robot switches to slithering gait to move to the target in Fig. 11(b), at $t=10 \mathrm{~s}$. During this process, the sensor data is transmitted to the SpiNNaker board, where calculates the relative bias between the robot and the target every two seconds. Then the snake robot steers to the left according to the calculated bias in Fig. 11(c), at $t=20 \mathrm{~s}$. Finally, the robot reaches the target pole, as shown in Fig. 11(d), at $t=35 \mathrm{~s}$. In summary, the target observing and tracking is achieved autonomously by the snake-like robot under the proposed slithering gait. 

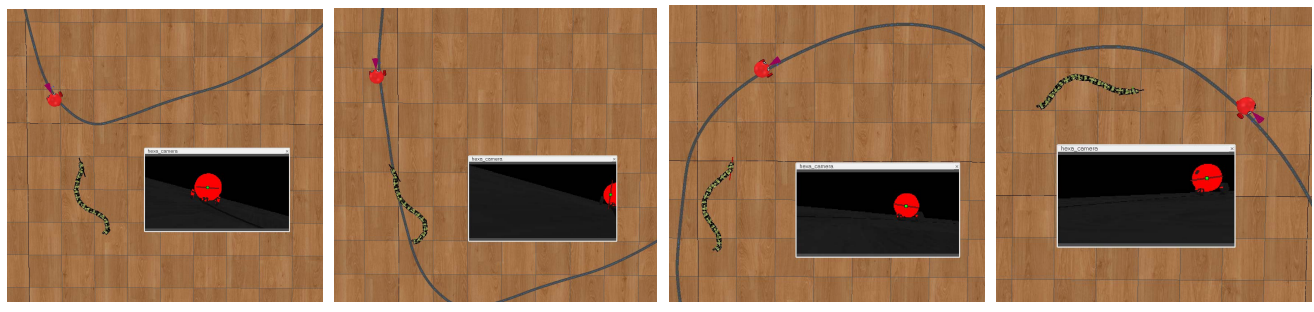

Fig. 10: Montage of the simulation scenario: The snake robot tracks a red car moving along the black path. The visual image is shown in the top right of each figure. The red spot means the relative position of the car in the robot's view field.

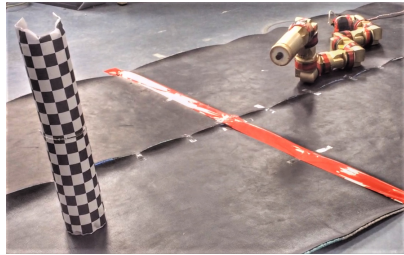

(a) Search for the target, $\mathrm{t}=5 \mathrm{~s}$

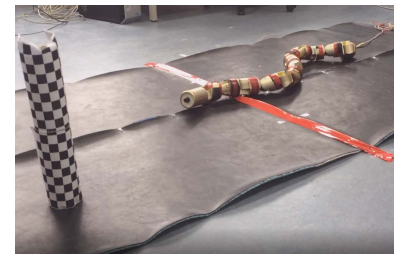

(b) Starts to slither, $\mathrm{t}=10 \mathrm{~s}$

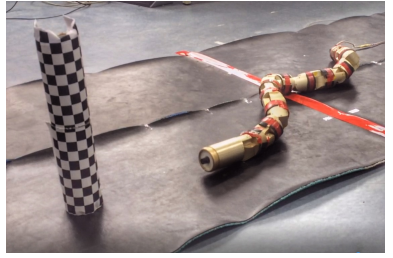

(c) Move to the left, $\mathrm{t}=20 \mathrm{~s}$

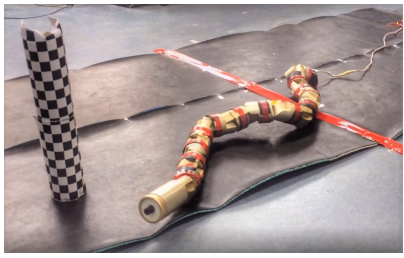

(d) Approach the target, $\mathrm{t}=35 \mathrm{~s}$

Fig. 11: Montage of the snake-like robot slithering to the target pole. The forward speed is approximately $0.15 \mathrm{~m} / \mathrm{s}$.

\section{CONClusion AND Future Work}

We have designed a slithering gait for snake-like robot for the purpose of autonomous locomotion. With this gait, the robot can travel and meanwhile observe the environment in an efficient and stable way. The visual sensors mounted in the head module can ensure an acceptable swing for observation scenarios by adopting the linear amplitude reduction and head orientation compensation methods. Simulations and prototype experiments demonstrate the practicality and effectiveness of the slithering gait in autonomous locomotion scenarios. For future work, an optimization algorithm for head orientation will be investigated for different sets of gaits parameters. This could make the robot more adaptive to different implementation scenarios.

\section{ACKNOWLEDGEMENTS}

The research leading to these results has received funding from the European Union Research and Innovation Programme Horizon 2020 (H2020/2014-2020) under grant agreement No. 720270 (Human Brain Project, HBP) and the Chinese Scholarship Council.

\section{REFERENCES}

[1] P. Liljebäck, K. Y. Pettersen, O. Stavdahl, and J. T. Gravdahl, Snake robots: modelling, mechatronics, and control. Springer Science \& Business Media, 2012.

[2] M. Mori and S. Hirose, "Three-dimensional serpentine motion and lateral rolling by active cord mechanism acm-r3," in IEEE/RSJ International Conference on Intelligent Robots and Systems, 2002., vol. 1, 2002, pp. 829-834 vol.1.

[3] M. Tesch, K. Lipkin, I. Brown, R. Hatton, A. Peck, J. Rembisz, and H. Choset, "Parameterized and scripted gaits for modular snake robots," Advanced Robotics, vol. 23, no. 9, pp. 1131-1158, 2009.

[4] E. Kelasidi, K. Y. Pettersen, J. T. Gravdahl, and P. Liljebäck, "Modeling of underwater snake robots," in IEEE International Conference on Robotics and Automation (ICRA), 2014. IEEE, 2014, pp. 4540-4547.

[5] F. Galluppi, C. Denk, M. C. Meiner, T. C. Stewart, L. A. Plana, C. Eliasmith, S. Furber, and J. Conradt, "Event-based neural computing on an autonomous mobile platform," in IEEE International Conference on Robotics and Automation (ICRA), 2014. IEEE, 2014, pp. 2862-2867.
[6] M. M. Khan, D. R. Lester, L. A. Plana, A. Rast, X. Jin, E. Painkras, and S. B. Furber, "Spinnaker: mapping neural networks onto a massively-parallel chip multiprocessor," in Neural Networks, 2008. IJCNN 2008.(IEEE World Congress on Computational Intelligence). IEEE International Joint Conference on. Ieee, 2008, pp. 2849-2856.

[7] D. L. Hu and M. Shelley, "Slithering locomotion," in Natural locomotion in fluids and on surfaces. Springer, 2012, pp. 117-135.

[8] R. L. Hatton, R. A. Knepper, H. Choset, D. Rollinson, C. Gong, and E. Galceran, "Snakes on a plan: Toward combining planning and control," in IEEE International Conference on Robotics and Automation (ICRA), 2013. IEEE, 2013, pp. 5174-5181.

[9] X. Wu and S. Ma, "Neurally controlled steering for collision-free behavior of a snake robot," IEEE Transactions on Control Systems Technology, vol. 21, no. 6, pp. 2443-2449, 2013.

[10] M. Tanaka and K. Tanaka, "Shape control of a snake robot with joint limit and self-collision avoidance," IEEE Transactions on Control Systems Technology, 2016.

[11] K. Melo and L. Paez, "Experimental determination of control parameter intervals for repeatable gaits in modular snake robots," in 2014 IEEE International Symposium on Safety, Security, and Rescue Robotics (2014). IEEE, 2014, pp. 1-7.

[12] H. Ohno and S. Hirose, "Design of slim slime robot and its gait of locomotion," in IEEE/RSJ International Conference on Intelligent Robots and Systems., vol. 2, 2001, pp. 707-715.

[13] W. Zhen, C. Gong, and H. Choset, "Modeling rolling gaits of a snake robot," in IEEE International Conference on Robotics and Automation (ICRA), 2015. IEEE, 2015, pp. 3741-3746.

[14] J. W. Burdick, J. Radford, and G. S. Chirikjian, "A "sidewinding" locomotion gait for hyper-redundant robots," in IEEE International Conference on Robotics and Automation, 1993. IEEE, 1993, pp. 101-106.

[15] R. L. Hatton and H. Choset, "Sidewinding on slopes," in IEEE International Conference on Robotics and Automation (ICRA), 2010. IEEE, 2010, pp. 691-696.

[16] C. Gong, M. J. Travers, X. Fu, and H. Choset, "Extended gait equation for sidewinding," in IEEE International Conference on Robotics and Automation (ICRA), 2013. IEEE, 2013, pp. 5162-5167.

[17] C. Gong, R. L. Hatton, and H. Choset, "Conical sidewinding," in 2012 IEEE International Conference on Robotics and Automation, May 2012, pp. 4222-4227.

[18] C. Gong, M. J. Travers, H. C. Astley, L. Li, J. R. Mendelson, D. I. Goldman, and H. Choset, "Kinematic gait synthesis for snake robots," The International Journal of Robotics Research, vol. 35, no. 1-3, pp. 100-113, 2016.

[19] J. Lishman and D. Lee, "The autonomy of visual kinaesthesis," Perception, vol. 2, no. 3, pp. 287-294, 1973.

[20] K. J. Dowling, "Limbless locomotion: learning to crawl with a snake robot," Ph.D. dissertation, NASA, 1996. 